\title{
Oral proteasome inhibitor maintenance for multiple myeloma
}

Niels W.C.J. van de Donk $\mathrm{MD}^{1}$ and Kwee Yong $\mathrm{MD}^{2}$

${ }^{1}$ Amsterdam UMC, Vrije Universiteit Amsterdam, Department of Hematology, Amsterdam, The Netherlands

${ }^{2}$ Department of Haematology, University College London, London, United Kingdom

\section{Correspondence to:}

N.W.C.J. van de Donk, MD, PhD

Amsterdam UMC, Vrije Universiteit Amsterdam, Department of Hematology

De Boelelaan 1117, 1081 HV, Amsterdam, The Netherlands

Phone: $+31(0) 204442604$

Fax: $+31(0) 204442601$

Text word count: 700

Number of references: 12

Number of figures: 0

Number of tables: 0 
High-dose melphalan with autologous stem cell transplantation (auto-SCT) has significantly improved survival of $\mathrm{MM}$ patients ${ }^{1-3}$, however the majority of patients eventually experience relapse. Among strategies to improve outcomes after auto-SCT is the use of maintenance therapy to sustain disease response and delay relapse.

Thalidomide was the first novel agent evaluated as maintenance therapy. Although posttransplant thalidomide improves progression-free survival (PFS) and overall survival (OS) ${ }^{4}$, prolonged use is limited by peripheral neuropathy, the main cause of treatment discontinuation ${ }^{5}$. Lenalidomide, a more potent and less toxic second-generation immunomodulatory drug (IMiD), is currently the only agent approved for post-transplant maintenance, based on improved PFS with lenalidomide versus placebo or observation ${ }^{6-9}$. Moreover, lenalidomide maintenance also prolongs OS with a reduced risk of death by approximately $25 \%^{9,10}$. Prolonged use of lenalidomide, however, is associated with increased risk of cytopenias, diarrhea, thromboembolic events, and secondary primary malignancies $(\mathrm{SPMs})^{10}$. Furthermore, the efficacy in patients with high-risk cytogenetics remains a matter of debate with a meta-analysis showing no survival benefit in this patient subset ${ }^{10}$. In contrast, the Myeloma XI study showed improved OS irrespective of cytogenetic risk, but lenalidomide maintenance did not completely abrogate the inferior outcomes associated with adverse cytogenetic abnormalities ${ }^{9}$.

Importantly, the use of the proteasome inhibitor, bortezomib, for induction and as posttransplant maintenance improves outcomes of newly diagnosed MM patients, especially those with high-risk features such as del(17p $)^{11}$. Long-term bortezomib therapy, however, is limited by neurotoxicity, and the inconvenience of subcutaneous administration. Thus, the development of the oral proteasome inhibitor, ixazomib, represents an important advance.

In The Lancet, Meletios Dimopoulos and colleagues report the results of a randomized phase 3 study (TOURMALINE-MM3), in which newly diagnosed MM patients, treated with standard-of-care induction therapy followed by high-dose melphalan and auto-SCT, were randomly assigned to receive once-weekly ixazomib or placebo for 3 weeks out of a 4 week cycle for 26 cycles or until progression. Ixazomib maintenance improved depth of response, when compared to the placebo group. At a median follow-up of 31 months, median PFS in 
patients receiving ixazomib was 26.5 months, compared to 21.3 months in the placebo group, representing a $28 \%$ reduction in risk of death or progression. The PFS benefit of ixazomib maintenance was observed irrespective of cytogenetic risk or MRD status at study entry. Importantly, ixazomib maintenance was well tolerated with low rates of polyneuropathy, and without increased risk of thromboembolic events or SPMs. Although, thrombocytopenia and gastrointestinal adverse events were more common with ixazomib, these were largely grade $1 / 2$. This translated into a similar rate of treatment discontinuation and equivalent quality of life in the ixazomib and placebo groups.

Several questions, however, remain to be answered. Although direct comparisons of postauto-SCT maintenance studies are confounded by differences in patient populations and induction regimens, PFS benefit was more modest with ixazomib maintenance, when compared to studies with lenalidomide (median PFS in meta-analysis: 52.8 months in lenalidomide group versus 23.5 months in control group ${ }^{10}$ ). This difference in activity may be related to variability in treatment duration (fixed duration versus treatment until progression), or suboptimal ixazomib dose intensity. In addition, there are important biological differences between agents, and the immune stimulating effects of lenalidomide may contribute to long-term control of residual MM cells.

It is currently also unknown whether ixazomib maintenance leads to the development of drug-resistant relapses, or affects response to subsequent proteasome inhibitor-based treatment. Analysis of PFS2 and OS is important in this context, but this requires longer follow-up. Furthermore, in the absence of a head-to-head comparison between ixazomib and lenalidomide, it is currently unclear which specific subgroups may benefit from ixazomib maintenance. Ixazomib may be a new maintenance option for patients intolerant to lenalidomide, or those with high-risk disease. The good tolerability profile of ixazomib (discontinuation due to adverse events: $7 \%$ versus $29 \%$ with lenalidomide ${ }^{10}$ ), may also facilitate combinations with other anti-MM agents, for example, ongoing trials are evaluating ixazomib plus lenalidomide maintenance, which may be of particular value in high-risk disease ${ }^{12}$. In the future, MRD-assessments may help to identify patients for more intensive maintenance strategies, and also guide optimal treatment duration to reduce adverse events and financial toxicity. Altogether, it is expected that such new maintenance 
strategies will further improve outcomes in $\mathrm{MM}$, and the development of ixazomib as a maintenance agent is an important step in this direction.

\section{Conflict of Interest}

Niels van de Donk reports research support from Celgene, Janssen Pharmaceuticals, Novartis, BMS, and Amgen; advisory boards for Celgene, Janssen Pharmaceuticals, Novartis, BMS, AMGEN, Takeda, Bayer, and Servier.

Kwee Yong reports research support from Amgen, Celgene, Janssen Pharmaceuticals;

advisory boards for Janssen Pharmaceuticals, Novartis, Celgene, Amgen, Takeda, and Abbvie. 


\section{References}

1. Attal M, Lauwers-Cances V, Hulin C, et al. Lenalidomide, Bortezomib, and Dexamethasone with Transplantation for Myeloma. N Engl J Med 2017; 376(14): 1311-20.

2. Cavo M, Hajek R, Pantani L, et al. Autologous Stem Cell Transplantation Versus Bortezomib-Melphalan-Prednisone for Newly Diagnosed Multiple Myeloma: Second Interim Analysis of the Phase 3 EMN02/HO95 Study. Blood 2017; 130 (suppl 1): 397.

3. Gay F, Oliva S, Petrucci MT, et al. Chemotherapy plus lenalidomide versus autologous transplantation, followed by lenalidomide plus prednisone versus lenalidomide maintenance, in patients with multiple myeloma: a randomised, multicentre, phase 3 trial. Lancet Oncol 2015; 16(16): 1617-29.

4. Ye X, Huang J, Pan Q, Li W. Maintenance therapy with immunomodulatory drugs after autologous stem cell transplantation in patients with multiple myeloma: a meta-analysis of randomized controlled trials. PloS one 2013; 8(8): e72635.

5. van de Donk NW, van der Holt B, Minnema MC, et al. Thalidomide before and after autologous stem cell transplantation in recently diagnosed multiple myeloma (HOVON-50): long-term results from the phase 3, randomised controlled trial. Lancet Haematol 2018; 5(10): e479-e92.

6. McCarthy PL, Owzar K, Hofmeister CC, et al. Lenalidomide after stem-cell transplantation for multiple myeloma. N Engl J Med 2012; 366(19): 1770-81.

7. Attal M, Lauwers-Cances V, Marit G, et al. Lenalidomide maintenance after stem-cell transplantation for multiple myeloma. N Engl J Med 2012; 366(19): 1782-91.

8. Palumbo A, Cavallo F, Gay F, et al. Autologous transplantation and maintenance therapy in multiple myeloma. N Engl J Med 2014; 371(10): 895-905.

9. Jackson G, Davies F, Pawlyn C, et al. Lenalidomide maintenance significantly improves outcomes compared to obervation irrespective of cytogenetic risk: results of the Myeloma XI trial Blood 2017; 130 (Suppl 1).

10. McCarthy PL, Holstein SA, Petrucci MT, et al. Lenalidomide Maintenance After Autologous Stem-Cell Transplantation in Newly Diagnosed Multiple Myeloma: A MetaAnalysis. J Clin Oncol 2017; 35(29): 3279-89.

11. Goldschmidt H, Lokhorst HM, Mai EK, et al. Bortezomib before and after high-dose therapy in myeloma: long-term results from the phase III HOVON-65/GMMG-HD4 trial. Leukemia 2018; 32(2): 383-90.

12. Patel KK, Shah J, Feng L, et al. Update on a Phase II Study of Ixazomib with Lenalidomide As Maintenance Therapy Following Autologous Stem Cell Transplant in Patients with Multiple Myeloma. Blood 2017; 130 (Suppl 1): 437. 